\title{
Atypical Vascular Proliferation Secondary to Radiotherapy in a Patient With a History of Synovial Sarcoma
}

\author{
Carlos E. Bonilla ${ }^{1}$, Lucy M. Perez Lugo ${ }^{2}$, Camilo Vallejo Yepes ${ }^{1}$, Handerson R. Osma Charris ${ }^{1}$ \\ 1. Oncology, Instituto Nacional De Cancerología, Bogota, COL 2. Internal Medicine, Instituto Nacional De \\ Cancerología, Bogota, COL
}

Corresponding author: Carlos E. Bonilla, carlosbonillamd@gmail.com

\begin{abstract}
We present the case of a 21-year-old male patient with a history of monophasic synovial sarcoma in his left thigh, which was treated with surgical resection, radiotherapy, and chemotherapy with mesna, doxorubicin, and Ifosfamide (MAI protocol). Approximately six years after the end of the oncological treatment, he presented a nodular, polypoid lesion in the left popliteal region, which was painless and fast growing. Ultimately, the biopsy was consistent with atypical vascular proliferation (AVP). Vascular lesions after radiotherapy include a wide spectrum of pathologies that range from benign lesions such as AVP to malignant ones with very poor prognosis such as angiosarcoma, the distinction between one and the other can be difficult, being the determination rearrangement or amplification of gene c-myc, a key to make an accurate diagnosis in case of doubt.
\end{abstract}

Received 08/25/2020

Review began 08/28/2020 Review ended 08/28/2020 Published 09/01/2020

(c) Copyright 2020

Bonilla et al. This is an open access article distributed under the terms of the Creative Commons Attribution License CC-BY 4.0., which permits unrestricted use, distribution, and reproduction in any medium, provided the original author and source are credited.
Categories: Dermatology, Pathology, Oncology

Keywords: c-myc, synovial sarcoma, angiogenesis, cutaneous vascular neoplasms, angiosarcoma, atypical vascular proliferation

\section{Introduction}

An atypical vascular lesion is an unusual complication following treatment with radiation therapy for breast cancer and other diseases [1]. It covers a spectrum of diseases ranging from benign lesions such as atypical vascular proliferation (AVP) to truly malignant entities such as angiosarcoma [2]. Occasionally, it can be difficult to distinguish histologically between these entities so is necessary to rely on molecular studies such as the amplification of c-myc gene, this test can be positive in up to $71 \%$ of angiosarcoma cases [1-3]. We report the case of a patient with AVP following treatment with adjuvant radiation therapy for a left lower extremity synovial sarcoma, treated with surgical resection, subsequently without relapse episodes to date; determination of rearrangements or amplifications of the c-myc gene was vital to rule out angiosarcoma, identify prognosis and, therefore, there was no need for additional therapies.

\section{Case Presentation}

A 21-year-old male patient with a history of stage III monophasic synovial sarcoma in the left thigh (pT2bNOM0) in 2012 was treated with surgical resection, followed by adjuvant radiotherapy with 6600 cGY in 33 fractions and four sequential chemotherapy cycles with mesna, doxorubicin and ifosfamide (MAI protocol). In 2018, he presented a painless polypoid lesion in the left popliteal fossa region with rapid growth, reaching $1.5 \mathrm{~cm}$ in diameter (Figure 1). An excisional biopsy was performed and histopathological analysis reported a vascular proliferation with immunohistochemistry positive for cluster of differentiation 31 (CD31), negative for human herpesvirus 8 (HHV8), 20\% Ki67 and focal areas of stroma of necrotic aspect, suggesting a diagnosis of AVP versus angiosarcoma (Figure 2). Due to the diagnostic uncertainty, fluorescence in situ hybridization (FISH) of the c-myc gene was performed with negative results, confirming the diagnosis of AVP and reasonably ruling out the possibility of secondary angiosarcoma. At the clinical follow-up in 2020, after two years of resection of the vascular lesion, the patient remains free of relapses. 


\section{Cureus}
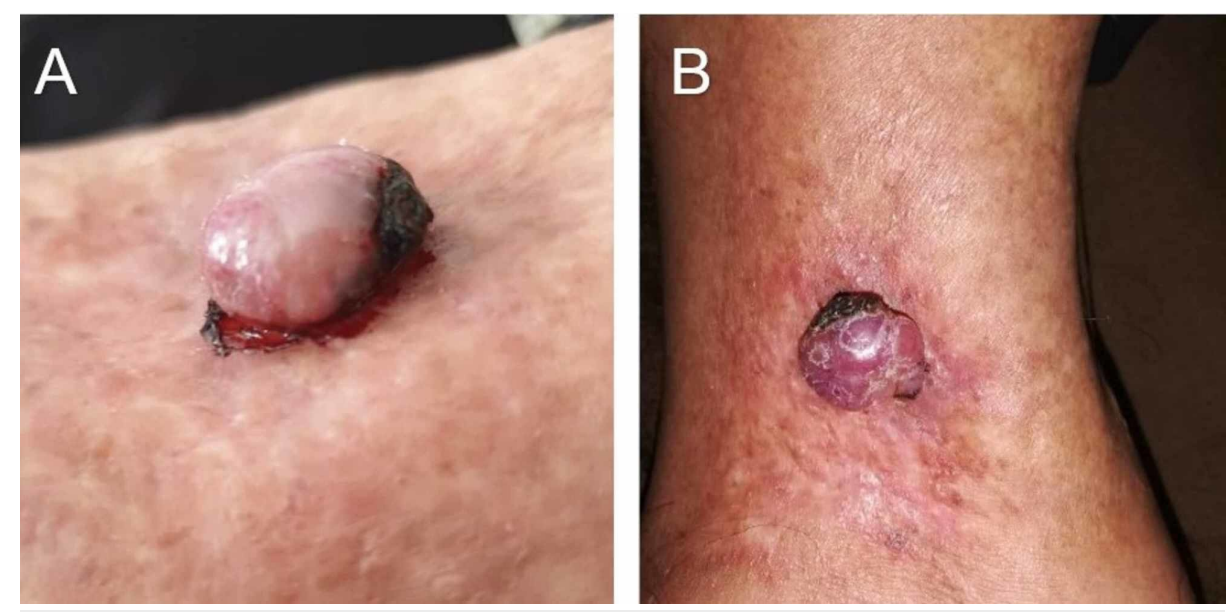

\section{FIGURE 1: Nodular lesion}

A. Lateral view of patient's multilobulated polypoid lesion with eccentric necrotic area that appeared six years after radiotherapy. B. Superior view of the same lesion.
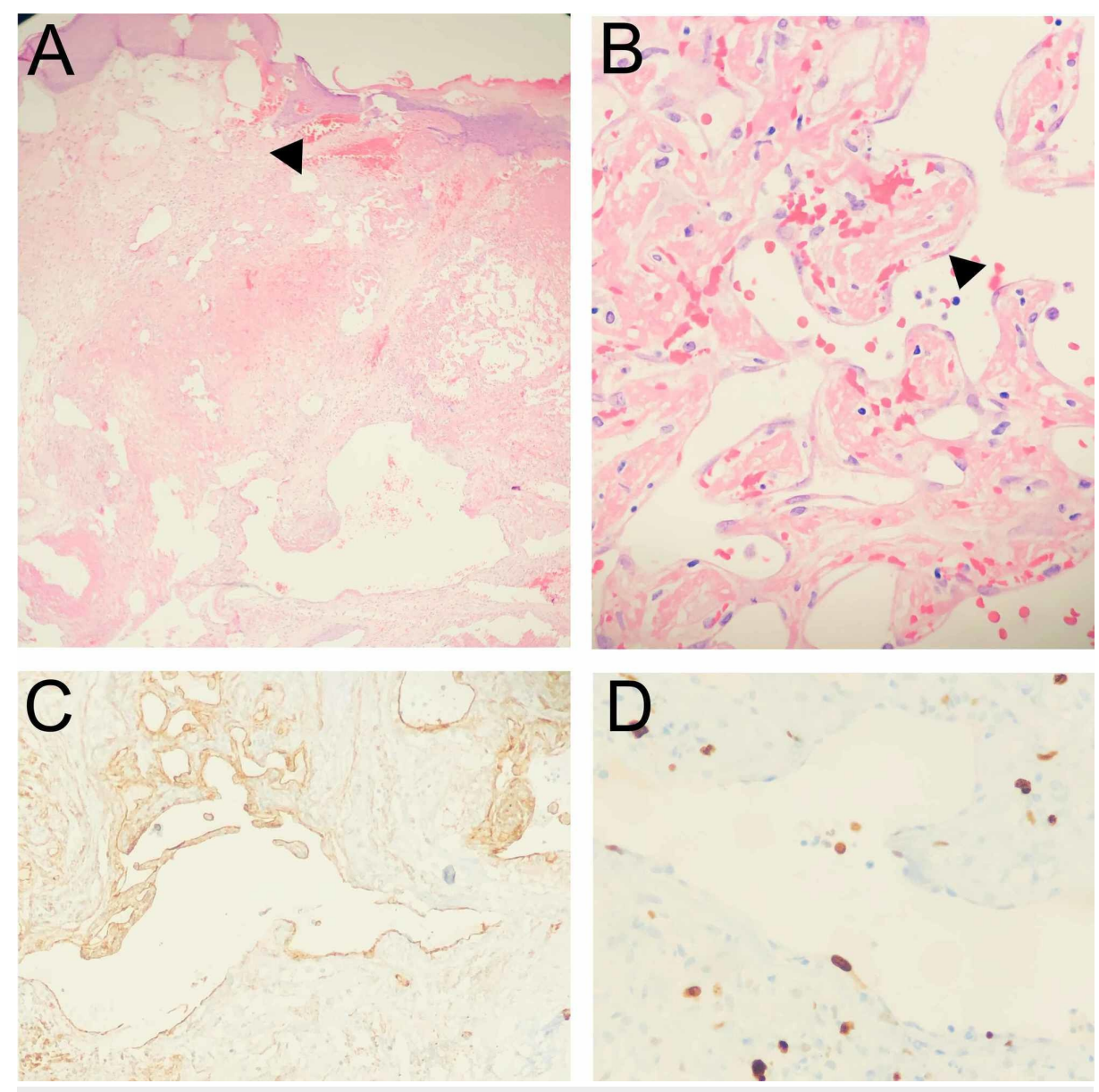

\section{FIGURE 2: Histopathological assessment}

A: Skin with acanthosis and atypical vascular proliferation at dermis level made up of dilated, thin-walled congestive vessels with reactive-looking endothelium is observed in the hematoxylin-eosin (HE)-stained cuts (black arrow). B: There are also vascular areas constituted by papillary projections with hyalinized centers and focal areas of stroma of necrotic aspect (black arrow). C: This vascular proliferation shows

immunoreactivity for the immunohistochemical markers, cluster of differentiation 31 (CD31) and FLI1 (in a focal way). D: There is negativity for the latent nuclear antigen-1 (LNA1) and human herpesvirus 8 (HHV8). Little cell proliferation is observed when measured with Ki67. 


\section{Cureus}

\section{Discussion}

AVP is a rare complication after treatment with radiotherapy [4], usually described after treatment of breast and other gynecological cancers [4,5], and less frequently in other types of neoplasms [6-9]. This complication is included in a spectrum of vascular entities in previously treated sites that includes benign lesions, atypical proliferation, and finally angiosarcoma $[2,10,11]$. They usually occur three-to-six years after treatment with radiation therapy [5,12]; however, there are reports of cases as early as eight months [13]. Benign lymphangiomatous lesions usually present as well-circumscribed, solitary papules that in histopathology show no atypia or proliferative activity. In AVP, the usual clinical presentation includes the presence of papules with coloring ranging from red to brown, most of them smaller than $20 \mathrm{~mm}[14,15]$. The appearance of up to four synchronous lesions has been reported, however, the most common form of presentation is the isolated presence of a single lesion as described by Brenn et al. in a series of 42 cases [16].

The term atypical vascular lesion or proliferation was introduced by Rosen and Fineberg in 1994 in a series of cases in which they described the similarities and differences with angiosarcoma [17,18]. AVP usually have a benign course, without fatal complications or secondary metastatic staining [14]. It is described more frequently in women, probably because the pathology in which it has been found most often is posterior to the treatment of breast cancer. In the review of 193 cases of vascular proliferation by Zhong et al., the condition in men corresponded to only $1.6 \%$ of cases [6].

Since it shares some similarities with secondary angiosarcoma histologically [1] but with a different prognosis and management approach, it is important to make a diagnosis with certainty. Table 1 describes some of the histopathological characteristics that allow this distinction to be made.

\begin{tabular}{|c|c|c|}
\hline Histopathological characteristic & Atypical vascular proliferation & Angiosarcoma \\
\hline Subcutaneous infiltration & - & +++ \\
\hline Papillary endothelial hyperplasia & - & +++ \\
\hline Protruding nucleoli & - & +++ \\
\hline Mitotic figures & - & +++ \\
\hline Significant cytological atypia & - & +++ \\
\hline Blood lakes & - & ++ \\
\hline Dermal collagen dissection & \pm & +++ \\
\hline Anastomotic cups & ++ & +++ \\
\hline Hyperchromic endothelial cells & +++ & ++ \\
\hline Chronic inflammation & +++ & + \\
\hline Relatively circumscribed & +++ & - \\
\hline Projection of the stroma to the lumen & +++ & - \\
\hline Cluster of differentiation (CD)34 & ++ & + \\
\hline CD31 & +++ & +++ \\
\hline D2-40 & ++ & + \\
\hline MYC expression & - & +++ \\
\hline P53 & ++ & ++ \\
\hline Prox1 & $-/+$ & +++ \\
\hline
\end{tabular}

TABLE 1: Histopathological characteristics that differentiate angiosarcoma from atypical vascular proliferation [17,18].

Although some histopathologic features may help differentiate AVP from angiosarcoma, they may have common characteristics that make diagnosis difficult. The myc genes are a family of proto-oncogenes including c-myc, L-myc, and N-myc, with c-myc being the most widely used. This gene has three exons located on chromosome 8q24 which plays an important role in cell division, growth and apoptosis [19]. FISH 
analysis for c-myc rearrangement is used to distinguish atypical vascular lesions from angiosarcoma, with positive rearrangement or amplification in cases of radiation-supported angiosarcoma and negative in atypical vascular lesions [1-3].

The clinical behavior of the AVP is benign, and to date no cases of progression or death associated with the condition have been reported. However, new lesions in the same field of irradiation with potential expression of c-myc and progression to angiosarcoma may occur during follow-up [16]. The treatment of choice is complete surgical resection of the lesion, and if new lesions appear in the radiation field, a new biopsy should be performed [20].

\section{Conclusions}

AVP is a rare post-radiotherapy skin complication, usually described after breast cancer treatment and only rarely in other pathologies. The histopathological study of vascular lesion after radiotherapy is important, because the spectrum of pathologies includes frankly benign entities, AVP, and finally angiosarcoma, which differ in prognosis and treatment. The use of immunofluorescence techniques to evaluate c-myc rearrangement or amplification is useful in cases with diagnostic doubt.

\section{Additional Information}

\section{Disclosures}

Human subjects: Consent was obtained by all participants in this study. Conflicts of interest: In compliance with the ICMJE uniform disclosure form, all authors declare the following: Payment/services info: All authors have declared that no financial support was received from any organization for the submitted work. Financial relationships: All authors have declared that they have no financial relationships at present or within the previous three years with any organizations that might have an interest in the submitted work. Other relationships: All authors have declared that there are no other relationships or activities that could appear to have influenced the submitted work.

\section{References}

1. Feller JK, Mahalingam M: C-myc and cutaneous vascular neoplasms. Am J Dermatopathol. 2013, 35:364-9. 10.1097/DAD.0b013e31827aad83

2. Mentzel T, Schildhaus HU, Palmedo G, Büttner R, Kutzner H: Postradiation cutaneous angiosarcoma after treatment of breast carcinoma is characterized by MYC amplification in contrast to atypical vascular lesions after radiotherapy and control cases: clinicopathological, immunohistochemical and molecular analysis of 66 cases. Mod Pathol. 2012, 25:75-85. 10.1038/modpathol.2011.134

3. Guo T, Zhang L, Chang NE, Singer S, Maki RG, Antonescu CR: Consistent MYC and FLT4 gene amplification in radiation-induced angiosarcoma but not in other radiation-associated atypical vascular lesions. Genes Chromosomes Cancer. 2011, 50:25-33. 10.1002/gcc.20827

4. Mandrell J, Mehta S, McClure S: Atypical vascular lesion of the breast. J Am Acad Dermatol. 2010, 63:33740. 10.1016/j.jaad.2009.08.017

5. Gengler C, Coindre JM, Leroux A, et al.: Vascular proliferations of the skin after radiation therapy for breast cancer: clinicopathologic analysis of a series in favor of a benign process. Cancer. 2007, 109:1584-98. 10.1002/cncr.22586

6. Zhong CS, Raut CP, Glynn RJ, Nambudiri VE: Characteristics of atypical post-radiation vascular proliferation: a retrospective review of 193 patients. J Am Acad Dermatol. 2019, 10.1016/j.jaad.2019.10.036

7. Mudaliar KM, Borrowdale R, Mehrotra S: Post-radiation atypical vascular lesion/angiosarcoma arising in the larynx. Head Neck Pathol. 2014, 8:359-63. 10.1007/s12105-013-0511-7

8. Antonescu C: Malignant vascular tumors-an update. Mod Pathol. 2014, 27:S30-S38. 10.1038/modpathol.2013.176

9. Tomkinson C, Lu JQ: Meningioangiomatosis: a review of the variable manifestations and complex pathophysiology. J Neurol Sci. 2018, 392:130-6. 10.1016/j.jns.2018.07.018

10. Suarez A, Johnson-Jahangir H, Desman G, Avarbock A: Post-radiation atypical vascular proliferation on the head of a young woman: a diagnostic challenge. Dermatol Online J. 2015, 21:

11. Meattini I, Santi R, Scartoni D, et al.: Multiple cutaneous angiosarcomas after breast conserving surgery and bilateral adjuvant radiotherapy: an unusual case and review of the literature. Case Rep Oncol Med. 2014, 413030. 10.1155/2014/413030

12. Patton KT, Deyrup AT, Weiss SW: Atypical vascular lesions after surgery and radiation of the breast: a clinicopathologic study of 32 cases analyzing histologic heterogeneity and association with angiosarcoma. Am J Surg Pathol. 2008, 32:943-50. 10.1097/pas.0b013e31815bf8fe

13. Losch A, Chilek KD, Zirwas MJ: Post-radiation atypical vascular proliferation mimicking angiosarcoma eight months following breast-conserving therapy for breast carcinoma. J Clin Aesthet Dermatol. 2011, 4:47-8.

14. Brenn T, Fletcher CDM: Postradiation vascular proliferations: an increasing problem. Histopathology. 2006, 48:106-14. 10.1111/j.1365-2559.2005.02293.x

15. Vergier B, Tisseau L: Mesenchymal tumors of the skin. Atypical vascular proliferation after radiotherapy [Article in French]. Ann Pathol. 2009, 29:399-402. 10.1016/j.annpat.2009.10.016

16. Brenn T, Fletcher CDM: Radiation-associated cutaneous atypical vascular lesions and angiosarcoma: clinicopothologic analysis of 42 cases. Am J Surg Pathol. 2005, 29:983-96.

10.1097/01.pas.0000156664.04955.83

17. Fineberg S, Rosen PP: Cutaneous angiosarcoma and atypical vascular lesions of the skin and breast after 


\section{Cureus}

radiation therapy for breast carcinoma. Am J Clin Pathol. 1994, 102:757-63. 10.1093/ajcp/102.6.757

18. Flucke U, Requena L, Mentzel T: Radiation-induced vascular lesions of the skin: an overview . Adv Anat Pathol. 2013, 20:407-15. 10.1097/PAP.0b013e3182a92e19

19. Patel JH, Loboda AP, Showe MK, Showe LC, McMahon SB: Analysis of genomic targets reveals complex functions of MYC. Nat Rev Cancer. 2004, 4:562-568. 10.1038/nrc1393

20. Lucas DR: Angiosarcoma, radiation-associated angiosarcoma, and atypical vascular lesion. Arch Pathol Lab Med. 2009, 133:1804-9. 10.1043/1543-2165-133.11.1804 